International Journal of Pure and Applied Mathematics

Volume 86 No. 3 2013, 537-552

ISSN: 1311-8080 (printed version); ISSN: 1314-3395 (on-line version)

url: http://www.ijpam.eu

doi: http://dx.doi.org/10.12732/ijpam.v86i3.8

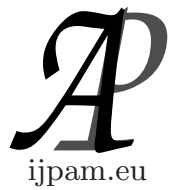

\title{
FUZZY CLOSURE SYSTEMS ON COMPLETE RESIDUATED LATTICES
}

\author{
Yong Chan Kim \\ Department of Mathematics \\ Gangneung-Wonju University \\ Gangneung, Gangwondo, 210-702, KOREA
}

\begin{abstract}
We investigate the properties of fuzzy closure systems on complete residuated lattices. In particular, we study the relations between fuzzy closure (interior) operators and fuzzy closure (interior) systems.
\end{abstract}

AMS Subject Classification: 03E72, 54A40,54B10

Key Words: fuzzy closure (interior) systems, complete residuated lattices, fuzzy closure (interior) operators

\section{Introduction}

The notion of closure systems and closure operators facilitated to study topological structures, logic and lattices. Recently, Bělohlávek [2] investigate the properties of fuzzy closure systems and fuzzy closure operators on residual lattices which supports part of foundation of theoretic computer science. Guo et.al [6] introduced fuzzy closure systems in a sense as the least upper bound on fuzzy partial ordered sets. It is a generalization of Bělohlávek's fuzzy closure system.

In this paper, we investigate the properties of fuzzy closure system with respect to the least upper bound on a complete residuated lattice. In particular, we study the relations between fuzzy closure (open) operators and fuzzy closure (interior) systems.

Received: April 12, 2013

(c) 2013 Academic Publications, Ltd. url: www.acadpubl.eu 


\section{Preliminaries}

Definition 1. (see [1-3], [6-9]) A structure $(L, \vee, \wedge, \odot, \rightarrow, \perp, \top)$ is called a complete residuated lattice if it satisfies the following conditions:

(R1) $(L, \vee, \wedge, \top, \perp)$ is a complete lattice with greatest element $\top$ and the least element $\perp$;

(R2) $(L, \odot, \top)$ is a commutative monoid;

(R3) it satisfies a residuation, i.e.

$$
a \odot b \leq c \text { iff } a \leq b \rightarrow c .
$$

Remark 2. (see [6-9]) A left-continuous t-norm $([0,1], \leq, \odot)$ defined by $a \rightarrow b=\bigvee\{c \mid a \odot c \leq b\}$ is a complete residuated lattice.

In this paper, we assume $(L, \wedge, \vee, \odot, \rightarrow, \top, \perp)$ is a complete residuated lattice with the law of double negation defined as $a=\left(a^{*}\right)^{*}$ where $a^{*}=a \rightarrow \perp$.

Lemma 3. (see [8]) For each $x, y, z, x_{i}, y_{i} \in L$, we have the following properties.

(1) If $y \leq z,(x \odot y) \leq(x \odot z), x \rightarrow y \leq x \rightarrow z$ and $z \rightarrow x \leq y \rightarrow x$.

(2) $x \rightarrow\left(\bigwedge_{i \in \Gamma} y_{i}\right)=\bigwedge_{i \in \Gamma}\left(x \rightarrow y_{i}\right)$ and $\left(\bigvee_{i \in \Gamma} x_{i}\right) \rightarrow y=\bigwedge_{i \in \Gamma}\left(x_{i} \rightarrow y\right)$.

(3) $(x \odot y) \rightarrow z=x \rightarrow(y \rightarrow z)$.

(4) $x \leq(x \rightarrow y) \rightarrow y$ and $x \odot(x \rightarrow y) \leq y$.

(5) $(x \rightarrow y) \odot(y \rightarrow z) \leq x \rightarrow z$.

(6) $(x \rightarrow z) \leq(x \odot y) \rightarrow(z \odot y)$.

(8) $(x \rightarrow y) \leq(y \rightarrow z) \rightarrow(x \rightarrow z)$.

(9) $(x \rightarrow y) \leq(z \rightarrow x) \rightarrow(z \rightarrow y)$.

(10) $x \rightarrow y=\top$ iff $x \leq y$.

(11) $x \rightarrow y=y^{*} \rightarrow x^{*}$.

(12) $(x \rightarrow y)^{*}=x \odot y^{*}$.

(13) $\bigwedge_{i \in \Gamma} x_{i}^{*}=\left(\bigvee_{i \in \Gamma} x_{i}\right)^{*}$ and $\bigvee_{i \in \Gamma} x_{i}^{*}=\left(\bigwedge_{i \in \Gamma} x_{i}\right)^{*}$.

Definition 4. Let $X$ be a set. A function $e_{X}: X \times X \rightarrow L$ is called a fuzzy partial order if it satisfies the following conditions :

(O1) $e_{X}(x, x)=\top$ for all $x \in X$, 
(O2) If $e_{X}(x, y)=e_{X}(y, x)=\top$, then $x=y$,

(O3) $e_{X}(x, y) \odot e_{X}(y, z) \leq e_{X}(x, z)$, for all $x, y, z \in X$.

The pair $\left(X, e_{X}\right)$ is called a fuzzy partial ordered se.

Example 5. (1) We define a function $e_{L}: L \times L \rightarrow L$ as

$$
e_{L}(x, y)=x \rightarrow y
$$

By Lemma $3(5),\left(L, e_{L}\right)$ is a fuzzy partial ordered set.

(2) We define a function $e_{L^{X}}: L^{X} \times L^{X} \rightarrow L$ as

$$
e_{L^{X}}(A, B)=\bigwedge_{x \in X}(A(x) \rightarrow B(x))
$$

By Lemma $3(5),\left(L^{X}, e_{L^{X}}\right)$ is a fuzzy partial ordered set.

\section{Fuzzy Closure Systems on Residuated Lattices}

Definition 6. (see [6]) Let $\left(X, e_{X}\right)$ be a fuzzy partial ordered set. A subset $M \subset X$ is called a fuzzy closure system if for any $x \in X$, there exists $m_{x} \in M$ such that

(M1) $e_{X}\left(x, m_{x}\right)=\top$ for all $x \in X$,

(M2) $e_{X}\left(n, m_{x}\right) \odot e_{X}(x, m) \leq e_{X}(n, m)$, for all $m, n \in M$.

We call $m_{x}$ the least upper bound of $x$ in $M$.

Remark 7. In the above definition, if $m_{x}^{1}$ and $m_{x}$ are the least upper bounds of $x$, then, by (M2), $\top=e_{X}\left(m_{x}^{1}, m_{x}^{1}\right) \odot e_{X}\left(x, m_{x}\right) \leq e_{X}\left(m_{x}^{1}, m_{x}\right)$ and $\top=e_{X}\left(m_{x}, m_{x}\right) \odot e_{X}\left(x, m_{x}^{1}\right) \leq e_{X}\left(m_{x}, m_{x}^{1}\right)$;i.e. $e_{X}\left(m_{x}^{1}, m_{x}\right)=e_{X}\left(m_{x}, m_{x}^{1}\right)=$ $\top$. Hence $m_{x}^{1}=m_{x}$. Thus the least upper bound $m_{x}$ is unique.

Theorem 8. Let $\mathcal{M}=\left\{A_{i} \mid i \in \Gamma\right\}$ be a subset of $L^{X}$. The following statement are equivalent:

(1) $\mathcal{M}=\left\{A_{i} \mid i \in \Gamma\right\}$ is a fuzzy closure system on $L^{X}$.

(2) For any $A \in L^{X}$, there exists $m_{A}=\bigwedge_{i \in \Gamma}\left(e_{L^{X}}\left(A, A_{i}\right) \rightarrow A_{i}\right) \in \mathcal{M}$.

$(3) \bigwedge_{i \in \Gamma}\left(a_{i} \rightarrow A_{i}\right) \in \mathcal{M}$. 
Proof. $(1) \Rightarrow(2)$. For each $A \in L^{X}$ and $m_{A} \in \mathcal{M}$, we will show that $m_{A}=\bigwedge_{i \in \Gamma^{X}}\left(e_{L^{X}}\left(A, A_{i}\right) \rightarrow A_{i}\right)$. Since $m_{A} \in \mathcal{M}$ and $e_{L^{X}}\left(A, m_{A}\right)=\top$, we have

$$
\bigwedge_{i \in \Gamma}\left(e_{L^{X}}\left(A, A_{i}\right) \rightarrow A_{i}\right) \leq e_{L^{X}}\left(A, m_{A}\right) \rightarrow m_{A}(x)=m_{A}(x) .
$$

Since $e_{L^{X}}\left(A, A_{i}\right) \odot e_{L^{X}}\left(A_{j}, m_{A}\right) \leq e_{L^{X}}\left(A_{j}, A_{i}\right)$ from $(\mathrm{M} 2)$ and $m_{A} \in \mathcal{M}$, put $A_{j}=m_{A}$, then

$$
\begin{aligned}
e_{L^{X}}\left(A, A_{i}\right) & =e_{L^{X}}\left(A, A_{i}\right) \odot e_{L^{X}}\left(m_{A}, m_{A}\right) \\
& \leq e_{L^{X}}\left(m_{A}, A_{i}\right) \leq m_{A}(x) \rightarrow A_{i}(x) .
\end{aligned}
$$

Then $m_{A}(x) \leq e_{L^{X}}\left(m_{A}, A_{i}\right) \rightarrow A_{i}(x)$. Hence $m_{A}=\bigwedge_{i \in \Gamma}\left(e_{L^{X}}\left(A, A_{i}\right) \rightarrow A_{i}\right)$.

$$
\begin{aligned}
& (2) \Rightarrow(1) \text {. For any } A \in L^{X} \text {, put } m_{A}=\bigwedge_{i \in \Gamma}\left(e_{L^{X}}\left(A, A_{i}\right) \rightarrow A_{i}\right) . \\
& \begin{aligned}
e_{L^{X}}\left(A, m_{A}\right)= & e_{L^{X}}\left(A, \bigwedge_{i \in \Gamma}\left(e_{L^{X}}\left(A, A_{i}\right) \rightarrow A_{i}\right)\right) \\
= & \bigwedge_{x \in X}\left(A(x) \rightarrow \bigwedge_{i \in \Gamma^{\prime}}\left(e_{L^{X}}\left(A, A_{i}\right) \rightarrow A_{i}(x)\right)\right) \\
= & \bigwedge_{x \in X} \bigwedge_{i \in \Gamma}\left(A(x) \rightarrow\left(e_{L^{X}}\left(A, A_{i}\right) \rightarrow A_{i}(x)\right)\right) \\
= & \bigwedge_{x \in X} \bigwedge_{i \in \Gamma}\left(e_{L^{X}}\left(A, A_{i}\right) \rightarrow\left(A(x) \rightarrow A_{i}(x)\right)\right) \\
= & \bigwedge_{i \in \Gamma}\left(e_{L^{X}}\left(A, A_{i}\right) \rightarrow \bigwedge_{x \in X}\left(A(x) \rightarrow A_{i}(x)\right)\right) \\
= & \top . \\
& \left.\quad \leq e_{L^{X}}\left(A, A_{i}\right) \odot \bigwedge_{i \in \Gamma}\left(e_{L^{X}}\left(A, A_{i}\right) \rightarrow A_{i}\right)(x)\right) \\
& \leq e_{L^{X}}\left(A, A_{i}\right) \odot\left(e_{L^{X}}\left(A, A_{i}\right) \rightarrow A_{i}(x)\right) \leq A_{i}(x) .
\end{aligned}
\end{aligned}
$$

Hence $e_{L^{X}}\left(A, A_{i}\right) \leq m_{A}(x) \rightarrow A_{i}(x)$. Thus, $e_{L^{X}}\left(A, A_{i}\right) \leq e_{L^{X}}\left(m_{A}, A\right)$.

Therefore

$$
\begin{gathered}
e_{L^{X}}\left(A, A_{i}\right) \odot e_{L^{X}}\left(A_{j}, m_{A}\right) \leq e_{L^{X}}\left(m_{A}, A_{i}\right) \odot e_{L^{X}}\left(A_{j}, m_{A}\right) \leq e_{L^{X}}\left(A_{j}, A_{i}\right) . \\
(2) \Rightarrow(3) \text { Put } A=\bigwedge_{i \in \Gamma}\left(a_{i} \rightarrow A_{i}\right) . \text { Then } A \leq e_{L^{X}}\left(A, A_{i}\right) \rightarrow A_{i} \text { from }
\end{gathered}
$$
Lemma 3(4). Thus

$$
A \leq \bigwedge_{i \in \Gamma}\left(e_{L^{X}}\left(A, A_{i}\right) \rightarrow A_{i}\right)
$$

Since $\bigwedge_{i \in \Gamma}\left(a_{i} \rightarrow A_{i}\right) \odot a_{i} \leq\left(a_{i} \rightarrow A_{i}\right) \odot a_{i} \leq A_{i}$, then

$$
e_{L^{X}}\left(A, A_{i}\right)=e_{L^{X}}\left(\bigwedge_{i \in \Gamma}\left(a_{i} \rightarrow A_{i}\right), A_{i}\right) \geq a_{i}
$$


Thus

$$
\left(e_{L^{X}}\left(A, A_{i}\right) \rightarrow A_{i}\right) \odot a_{i} \leq\left(e_{L^{X}}\left(A, A_{i}\right) \rightarrow A_{i}\right) \odot e_{L^{X}}\left(A, A_{i}\right) \leq A_{i}
$$

Hence $e_{L^{X}}\left(A, A_{i}\right) \rightarrow A_{i} \leq a_{i} \rightarrow A_{i}$. Hence $\bigwedge_{i \in \Gamma}\left(e_{L^{X}}\left(A, A_{i}\right) \rightarrow A_{i}\right) \leq A$. It follows $\bigwedge_{i \in \Gamma}\left(e_{L^{X}}\left(A, A_{i}\right) \rightarrow A_{i}\right)=A \in \mathcal{M}$.

$(3) \Rightarrow(2)$ Put $a_{i}=e_{L^{X}}\left(A, A_{i}\right)$, it easily proved.

Remark 9. The condition in Theorem 8(2) is the fuzzy closure system defined by Bělohlávek in [2].

Definition 10. (see [6]) Let $\left(X, e_{X}\right)$ be a fuzzy partial ordered set. A subset $K \subset X$ is called a fuzzy interior system if for any $x \in X$, there exists $k_{x} \in K$ such that:

(K1) $e_{X}\left(k_{x}, x\right)=\top$ for all $x \in X$,

$(\mathrm{K} 2) e_{X}(m, x) \odot e_{X}\left(k_{x}, n\right) \leq e_{X}(m, n)$, for all $m, n \in K$.

We call $k_{x}$ the greatest lower bound of $x$ in $K$.

Remark 11. In the above definition, by a similar method as Remark 7, the greatest lower bound $k_{x}$ is unique.

Theorem 12. Let $\mathcal{K}=\left\{A_{i} \mid i \in \Gamma\right\}$ be a subset of $L^{X}$. The following statement are equivalent:

(1) $\mathcal{K}$ is a left interior system on $L^{X}$.

(2) For any $A \in L^{X}, \bigvee_{i \in \Gamma}\left(e_{L^{X}}\left(A_{i}, A\right) \odot A_{i}\right) \in \mathcal{K}$.

(3) For any $a_{i} \in L, \bigvee_{i \in \Gamma}\left(a_{i} \odot A_{i}\right) \in \mathcal{K}$. $\mathcal{M}$

Proof. $(1) \Rightarrow(2)$. We show that $k_{A}=\bigvee_{i \in \Gamma}\left(e_{L^{X}}\left(A_{i}, A\right) \odot A_{i}\right)$. Since $k_{A} \in$

$$
\bigvee_{i \in \Gamma}\left(e_{L^{X}}\left(A_{i}, A\right) \odot A_{i}(x)\right) \geq e_{L^{X}}\left(k_{A}, A\right) \odot k_{A}(x)=k_{A}(x)
$$

By $(\mathrm{K} 2)$, since $e_{L^{X}}\left(A_{i}, A\right) \odot e_{L^{X}}\left(k_{A}, A_{j}\right) \leq e_{L^{X}}\left(A_{i}, A_{j}\right)$ and $k_{A} \in \mathcal{K}$, put $A_{j}=$ $k_{A}$, then

$$
e_{L^{X}}\left(A_{i}, A\right)=e_{L^{X}}\left(k_{A}, k_{A}\right) \odot e_{L^{X}}\left(A_{i}, A\right) \leq e_{L^{X}}\left(A_{i}, k_{A}\right) .
$$

Hence $e_{L^{X}}\left(A_{i}, A\right) \leq A_{i}(x) \rightarrow k_{A}(x)$. Thus, $\bigvee_{i \in \Gamma}\left(e_{L^{X}}\left(A_{i}, A\right) \odot A_{i}(x)\right) \leq k_{A}(x)$. Therefore $k_{A}=\bigvee_{i \in \Gamma}\left(e_{L^{X}}\left(A_{i}, A\right) \odot A_{i}\right)$. 
$(2) \Rightarrow(1)$. For any $A \in L^{X}$, put $k_{A}=\bigvee_{i \in \Gamma}\left(e_{L^{X}}\left(A_{i}, A\right) \odot A_{i}\right)$.

$$
\begin{aligned}
e_{L^{X}}\left(k_{A}, A\right) & =e_{L^{X}}\left(\bigvee_{i \in \Gamma}\left(e_{L^{X}}\left(A_{i}, A\right) \odot A_{i} \rightarrow A\right)\right) \\
& =\bigwedge_{x \in X} \bigwedge_{i \in \Gamma}\left(e_{L^{X}}\left(A_{i}, A\right) \rightarrow\left(A_{i}(x) \rightarrow A(x)\right)\right) \\
& =\bigwedge_{i \in \Gamma}\left(e_{L^{X}}\left(A_{i}, A\right) \rightarrow \bigwedge_{x \in X}\left(A_{i}(x) \rightarrow A(x)\right)\right) \\
& =\bigwedge_{i \in \Gamma}\left(e_{L^{X}}\left(A_{i}, A\right) \rightarrow e_{L^{X}}\left(A_{i}, A\right)\right)=\top .
\end{aligned}
$$

Since $e_{L^{X}}\left(A_{i}, e_{L^{X}}\left(A, A_{i}\right) \odot A_{i}\right) \geq e_{L^{X}}\left(A_{i}, A\right)$, we have

$$
\begin{aligned}
e_{L^{X}}\left(A_{i}, k_{A}\right) & =e_{L^{X}}\left(A_{i}, \bigvee_{i \in \Gamma^{\prime}}\left(e_{L^{X}}\left(A_{i}, A\right) \odot A_{i}\right)\right. \\
& \geq e_{L^{X}}\left(A_{i}, e_{L^{X}}\left(A, A_{i}\right) \odot A_{i}\right) \geq e_{L^{X}}\left(A_{i}, A\right)
\end{aligned}
$$

Thus $e_{L^{X}}\left(A_{i}, A\right) \odot e_{L^{X}}\left(k_{A}, A_{j}\right) \leq e_{L^{X}}\left(A_{i}, k_{A}\right) \odot e_{L^{X}}\left(k_{A}, A_{j}\right) \leq e_{L^{X}}\left(A_{i}, A_{j}\right)$.

$(2) \Rightarrow(3)$ Put $A=\bigvee_{i \in \Gamma}\left(b_{i} \odot B_{i}\right)$. Then $A \geq e_{L^{X}}\left(B_{i}, A\right) \odot B_{i}$. Thus $A \geq$ $\bigvee_{i \in \Gamma}\left(e_{L^{X}}\left(B_{i}, A\right) \odot B_{i}\right)$. On the other hand, since $e_{L^{X}}\left(B_{i}, A\right)=e_{L^{X}}\left(B_{i}, \bigvee_{i \in \Gamma}\left(b_{i} \odot\right.\right.$ $\left.\left.B_{i}\right)\right) \geq b_{i}$, then $b_{i} \odot B_{i} \leq \bigvee_{i \in \Gamma}\left(e_{L^{X}}\left(B_{i}, A\right) \odot B_{i}\right)$. Hence $A \leq \bigvee_{i \in \Gamma}\left(e_{L^{X}}\left(B_{i}, A\right) \odot\right.$ $\left.B_{i}\right)$. It follows $\bigvee_{i \in \Gamma}\left(e_{L^{X}}\left(B_{i}, A\right) \odot B_{i}\right)=A \in \mathcal{K}$.

$(3) \Rightarrow(2)$ Put $b_{i}=e_{L^{X}}\left(B_{i}, A\right)$, it easily proved.

Theorem 13. Let $\left(X, e_{X}\right)$ be a fuzzy partial ordered set.

(1) If $(X, M)$ is a fuzzy closure system on $X$, then $(X, M)$ is a fuzzy interior system on $\left(X, e_{X}^{-1}\right)$ where $e_{X}^{-1}(x, y)=e_{X}(y, x)$.

(2) If $(X, K)$ is a fuzzy interior system on $X$, then $(X, K)$ is a fuzzy closure system on $\left(X, e_{X}^{-1}\right)$.

Proof. (1) $e_{X}^{-1}$ is a fuzzy partial order from:

$$
\begin{aligned}
& e_{X}^{-1}(x, y) \odot e_{X}^{-1}(y, z)=e_{X}(y, x) \odot e_{X}(z, y) \\
& \leq e_{X}(z, x)=e_{X}^{-1}(x, z) .
\end{aligned}
$$

Since $e_{X}\left(x, m_{x}\right)=\top$ and $e_{X}(x, m) \odot e_{X}\left(n, m_{x}\right) \leq e_{X}(n, m)$, for all $m, n \in M$, we have

$$
\begin{aligned}
& e_{X}^{-1}\left(m_{x}, x\right)=e_{X}\left(x, m_{x}\right)=\top \\
& e_{X}^{-1}(m, x) \odot e_{X}^{-1}\left(m_{x}, n\right)=e_{X}(x, m) \odot e_{X}\left(n, m_{x}\right) \\
& \leq e_{X}(n, m)=e_{X}^{-1}(m, n) .
\end{aligned}
$$

Hence $m_{x} \in M$ is the greatest upper bound of $x$. Thus $M$ is a fuzzy interior system on $\left(X, e_{X}^{-1}\right)$

(2) It is similarly proved. 
Theorem 14. Let $\left(L^{X}, e_{L^{X}}\right)$ be a fuzzy partial ordered set.

(1) If $\mathcal{M}$ is a fuzzy closure system on $L^{X}$, then $\mathcal{M}$ is a fuzzy interior system on $\left(L^{X}, e_{L^{X}}^{-1}\right)$ where

$$
e_{L^{X}}^{-1}(A, B)=e_{L^{X}}(B, A)=e_{L^{X}}\left(A^{*}, B^{*}\right) .
$$

In particular, put $\mathcal{K}_{M}=\left\{B^{*} \mid B \in \mathcal{M}\right\}$. Then $k_{D}=\left(m_{D^{*}}\right)^{*}$ is the greatest upper bound of $D$ on the fuzzy interior system $\mathcal{K}_{M}$.

(2) If $\mathcal{K}$ is a fuzzy interior system on $L^{X}$, then $\mathcal{K}$ is a fuzzy closure system on $\left(L^{X}, e_{L^{X}}^{-1}\right)$. In particular, put $\mathcal{M}_{K}=\left\{B^{*} \mid B \in \mathcal{K}\right\}$. Then $m_{A}=\left(k_{A^{*}}\right)^{*}$ is the least lower bound of $A$ on the fuzzy closure system $\mathcal{M}_{K}$.

Proof. (1) $e_{L^{X}}^{-1}(A, B)=e_{L^{X}}(B, A)=e_{L^{X}}\left(A^{*}, B^{*}\right)$ from:

$$
\begin{aligned}
e_{L^{X}}^{-1}(A, B) & =e_{L^{X}}(B, A)=\bigwedge_{x \in X}(B(x) \rightarrow A(x)) \\
& =\bigwedge_{x \in X}\left(A^{*}(x) \rightarrow B^{*}(x)\right)=e_{L^{X}}\left(A^{*}, B^{*}\right) .
\end{aligned}
$$

Since $e_{L^{X}}\left(\left(m_{A}\right)^{*}, A^{*}\right)=e_{L^{X}}\left(A, m_{A}\right)=\top$ and $e_{L^{X}}(A, B) \odot e_{L^{X}}\left(C, m_{A}\right) \leq$ $e_{L^{X}}(C, B)$, for all $B, C \in M$, we have

$$
\begin{aligned}
& e_{L^{X}}\left(\left(m_{A}\right)^{*}, A^{*}\right)=e_{L^{X}}^{-1}\left(m_{A}, A\right)=e_{L^{X}}\left(A, m_{A}\right)=\top, \\
& e_{L^{X}}\left(B^{*}, A^{*}\right) \odot e_{L^{X}}\left(\left(m_{A}\right)^{*}, C^{*}\right) \\
& =e_{L^{X}}^{-1}(B, A) \odot e_{L^{X}}^{-1}\left(m_{A}, C\right)=e_{L^{X}}(A, B) \odot e_{L^{X}}\left(C, m_{A}\right) \\
& \leq e_{L^{X}}(C, B)=e_{L^{X}}^{-1}(B, C)=e_{L^{X}}\left(B^{*}, C^{*}\right) .
\end{aligned}
$$

Put $A=D^{*}$. Since $A^{*}=\left(D^{*}\right)^{*}=D$, we have

$$
\begin{aligned}
& e_{L^{X}}\left(\left(m_{D^{*}}\right)^{*}, D\right)=\top, \\
& e_{L^{X}}\left(B^{*}, D\right) \odot e_{L^{X}}\left(\left(m_{D^{*}}\right)^{*}, C^{*}\right) \leq e_{L^{X}}\left(B^{*}, C^{*}\right) .
\end{aligned}
$$

Put $K_{M}=\left\{B^{*} \mid B \in \mathcal{M}\right\}$. Then $k_{D}=\left(m_{D^{*}}\right)^{*}$ is the greatest upper bound of $D$ on a fuzzy interior system $\left(X, K_{M}\right)$.

(2) Since $e_{L^{X}}\left(A^{*},\left(k_{A}\right)^{*}\right)=e_{L^{X}}\left(k_{A}, A\right)=\top$ and $e_{L^{X}}(B, A) \odot e_{L^{X}}\left(k_{A}, C\right) \leq$ $e_{X}(B, C)$, for all $B, C \in K$, we have

$$
\begin{aligned}
& e_{L^{X}}\left(A^{*},\left(k_{A}\right)^{*}\right)=e_{L^{X}}^{-1}\left(A, k_{A}\right)=e_{L^{X}}\left(k_{A}, A\right)=\top \\
& e_{L^{X}}\left(A^{*}, B^{*}\right) \odot e_{L^{X}}\left(C^{*},\left(k_{A}\right)^{*}\right) \\
& =e_{L^{X}}^{-1}(A, B) \odot e_{L^{X}}^{-1}\left(C, k_{A}\right)=e_{L^{X}}(B, A) \odot e_{L^{X}}\left(k_{A}, C\right) \\
& \leq e_{L^{X}}(B, C)=e_{L^{X}}^{-1}(C, B)=e_{L^{X}}\left(B^{*}, C^{*}\right) .
\end{aligned}
$$


Put $A=D^{*}$. Since $A^{*}=\left(D^{*}\right)^{*}=D$, we have

$$
\begin{aligned}
& e_{L^{X}}\left(D,\left(k_{D^{*}}\right)^{*}\right)=\top, \\
& e_{L^{X}}\left(D, B^{*}\right) \odot e_{L^{X}}\left(C^{*},\left(k_{D^{*}}\right)^{*}\right) \leq e_{L^{X}}\left(B^{*}, C^{*}\right) .
\end{aligned}
$$

Put $\mathcal{M}_{K}=\left\{B^{*} \mid B \in \mathcal{K}\right\}$. Then $m_{D}=\left(k_{D^{*}}\right)^{*}$ is the least lower bound of $D$ on a fuzzy closure system $\left(X, \mathcal{M}_{K}\right)$.

Definition 15. (see $[6,9])$ Let $\left(X, e_{X}\right)$ be a fuzzy partial ordered set. An operator $C: X \rightarrow X$ is called a fuzzy closure operator on $X$ if it satisfies the following conditions:

$(\mathrm{C} 1) e_{X}(x, C(x))=e_{X}(C(C(x)), C(x))=\top$,

(C2) $e_{X}(x, y) \leq e_{X}(C(x), C(y))$.

The pair $(X, C)$ is called a fuzzy closure space.

Definition 16. (see $[6,9]$ Let $\left(X, e_{X}\right)$ be a fuzzy partial ordered set. An operator $I: X \rightarrow X$ is called a fuzzy interior operator on $X$ if it satisfies the following conditions:

(I1) $e_{X}(I(x), x)=e_{X}(I(x), I(I(x)))=\top$,

(I2) $e_{X}(x, y) \leq e_{X}(I(x), I(y))$.

The pair $(X, I)$ is called a fuzzy interior space.

Theorem 17. Let $\left(X, e_{X}\right)$ be a fuzzy partial ordered set.

(1) Let $(X, I)$ be a fuzzy interior operator. Define $K_{I}=\{I(x) \mid x \in X\}$ and $K_{I}=\{I(x) \mid x \in X\}$. Then $\left(X, K_{I}\right)$ is a fuzzy interior system.

(2) Let $(X, K)$ be a fuzzy interior system. Define $I_{K}(x)=k_{x}$ where $I(x)$ is the greatest lower bound of $x$. Then $\left(X, I_{K}\right)$ is a fuzzy interior operator.

Proof. (1) Let $I$ be a fuzzy interior operator. Since $I(x) \in K_{I}$, then $e_{X}(I(x), x)=\top$. For $m \in K_{I}$, there exists $x_{m} \in X$ such that $I\left(x_{m}\right)=m$. Since $I$ is a fuzzy interior operator, $\top=e_{X}\left(m, x_{m}\right) \leq e_{X}\left(I(m), I\left(x_{m}\right)\right)=$ $e_{X}(I(m), m)$. Moreover, $e_{X}\left(I\left(x_{m}\right), I\left(I\left(x_{m}\right)\right)\right)=e_{X}(m, I(m))=\top$. Since

$$
e_{X}(I(m), m)=e_{X}(m, I(m))=\top
$$

$I(m)=m$. For any $m, n \in K_{I}$, we have $I(m)=m$ and $I(m)=m$. Thus

$$
\begin{aligned}
e_{X}(m, x) \odot e_{X}(I(x), n) & \leq e_{X}(I(m), I(x)) \odot e_{X}(I(x), n) \\
& \leq e_{X}(I(m), n)=e_{X}(m, n) .
\end{aligned}
$$


Hence $I(x)$ is the greatest lower bound of $x$. Thus $K_{I}$ be a fuzzy interior system.

(2) Let $K$ be a fuzzy interior system. Since $I_{K}(x)=k_{x}, e_{X}=\left(I_{K}(x), x\right)=$ $e_{X}\left(k_{x}, x\right)=\top$. For each $x_{1}, x_{2} \in X$,

$$
e_{X}\left(x_{1}, x_{2}\right)=e_{X}\left(I_{K}\left(x_{1}\right), x_{1}\right) \odot e_{X}\left(x_{1}, x_{2}\right) \leq e_{X}\left(I_{K}\left(x_{1}\right), x_{2}\right) .
$$

By (K2), since $e_{X}(m, x) \odot e_{X}\left(k_{x}, n\right) \leq e_{X}(m, n)$, for all $m, n \in K$, put $m=$ $I_{K}\left(x_{1}\right), x=x_{2}$ and $n=I_{K}\left(x_{2}\right)$, then $k_{x}=I_{K}\left(x_{2}\right)$ and

$$
e_{X}\left(I_{K}\left(x_{1}\right), x_{2}\right) \odot e_{X}\left(I_{K}\left(x_{2}\right), I_{K}\left(x_{2}\right)\right) \leq e_{X}\left(I_{K}\left(x_{1}\right), I_{K}\left(x_{2}\right)\right) .
$$

Hence $e_{X}\left(x_{1}, x_{2}\right) \leq e_{X}\left(I_{K}\left(x_{1}\right), I_{K}\left(x_{2}\right)\right)$. By $(\mathrm{K} 2), e_{X}(m, x) \odot e_{X}\left(k_{x}, n\right) \leq$ $e_{X}(m, n)$, for all $m, n \in K$. Since $I_{K}(x) \in K$, put $m=x=I_{K}(x)$ and $n=I_{K}\left(I_{K}(x)\right)$, then

$$
\top=e_{X}\left(I_{K}(x), I_{K}(x)\right) \odot e_{X}\left(I_{K}\left(I_{K}(x)\right), I_{K}\left(I_{K}(x)\right)\right) \leq e_{X}\left(I_{K}(x), I_{K}\left(I_{K}(x)\right)\right) .
$$

Hence $e_{X}\left(I_{K}(x), I_{K}\left(I_{K}(x)\right)\right)=\top$. Thus $I_{K}$ is a fuzzy interior operator.

Theorem 18. Let $\left(L^{X}, e_{L^{X}}\right)$ be a fuzzy partial ordered set.

(1) Let $\left(L^{X}, I\right)$ be a fuzzy interior operator. Define $K_{I}=\left\{I(A) \mid A \in L^{X}\right\}$. Then $\left(L^{X}, K_{I}\right)$ is a fuzzy interior system.

(2) Let $\left(L^{X}, K\right)$ is a fuzzy interior system. Define $I_{K}(A)=k_{A}$. Then $\left(L^{X}, I_{K}\right)$ is a fuzzy interior operator.

(3) In (1) and (2), $I_{K_{I}}=I$ and $K_{I_{K}}=K$.

Proof. (1) and (2) follow from Theorem 17.

(3) Since $K_{I}=\left\{I(A) \mid A \in L^{X}\right\}$, by Theorems 12(2) and 17(1),

$$
I_{K_{I}}(B)=\bigvee_{A \in L^{X}}\left(I(A) \odot e_{L^{X}}(I(A), B)\right)
$$

Since $e_{L^{X}}(I(B), B)=\top$,

$$
I_{K_{I}}(B) \geq I(B) \odot e_{L^{X}}(I(B), B)=I(B) .
$$

Since $I(A) \odot e_{L^{X}}(I(A), B) \leq B$, then

$$
B \geq I_{K_{I}}(B)=\bigvee_{A \in L^{X}}\left(I(A) \odot e_{L^{X}}(I(A), B)\right) \in K_{I},
$$


by $(\mathrm{K} 2)$,

$$
\top=e_{L^{X}}(I(B), I(B)) \odot e_{X}\left(I_{K_{I}}(B), B\right) \leq e_{X}\left(I_{K_{I}}(B), I(B)\right) .
$$

Hence $I_{K_{I}}(B) \leq I(B)$. Thus, $I_{K_{I}}(B)=I(B)$.

Let $K=\left\{A_{i} \mid i \in \Gamma\right\}$ be a fuzzy interior system. Then $I_{K}(A)=\bigvee_{i \in \Gamma}\left(A_{i} \odot\right.$ $\left.e_{L^{X}}\left(A, A_{i}\right)\right)$. Hence $K_{I_{K}}=\left\{\bigvee_{i \in \Gamma}\left(A_{i} \odot e_{L^{X}}\left(A_{i}, A\right)\right) \mid A \in L^{X}\right\}$. Let $A_{j} \in$ $K$. Then $\bigvee_{i \in \Gamma}\left(A_{i} \odot e_{L^{X}}\left(A_{i}, A_{j}\right)\right) \geq A_{j} \odot e_{L^{X}}\left(A_{j}, A_{j}\right)=A_{j}$. Since $A_{i} \odot$ $e_{L^{X}}\left(A_{i}, A_{j}\right) \leq A_{j}$, then $\bigvee_{i \in \Gamma}\left(A_{i} \odot e_{L^{X}}\left(A_{i}, A_{j}\right)\right) \leq A_{j}$. Thus

$$
\bigvee_{i \in \Gamma}\left(A_{i} \odot e_{L^{X}}\left(A_{i}, A_{j}\right)\right) \geq A_{j} \odot e_{L^{X}}\left(A_{j}, A_{j}\right)=A_{j} \in K_{I_{K}} .
$$

Let $B \in K_{I_{K}}$. By Theorem 12(2), $B \in K$. Hence $K_{I_{K}}=K$.

Corollary 19. Let $\left(X, e_{X}\right)$ be a fuzzy partial ordered set.

(1) Let $(X, C)$ be a fuzzy closure operator. Define $M_{C}=\{C(x) \mid x \in X\}$. Then $\left(X, M_{C}\right)$ is a fuzzy closure system.

(2) Let $(X, M)$ be a fuzzy closure system. Define $C_{M}(x)=m_{x}$. Then $\left(X, C_{M}\right)$ is a fuzzy closure operator.

Theorem 20. Let $\left(L^{X}, e_{L^{X}}\right)$ be a fuzzy partial ordered set.

(1) Let $\left(L^{X}, C\right)$ be a fuzzy closure operator. Define $M_{C}=\{C(A) \mid A \in$ $\left.L^{X}\right\}$. Then $\left(L^{X}, M_{C}\right)$ is a fuzzy closure system.

(2) Let $\left(L^{X}, M\right)$ be a fuzzy closure system. Define $C_{M}(A)=m_{A}$. Then $\left(L^{X}, C_{M}\right)$ is a fuzzy closure operator.

(3) In (1) and (2), $C_{M_{C}}=C$ and $M_{C_{M}}=M$.

Proof. (3) Since $M_{C}=\left\{C(A) \mid A \in L^{X}\right\}$, by Theorem 8(2),

$$
C_{M_{C}}(B)=\bigwedge_{A \in L^{X}}\left(e_{L^{X}}(B, C(A)) \rightarrow C(A)\right.
$$

Since $e_{L^{X}}(B, C(B))=\top$,

$$
C_{M_{C}}(B) \leq e_{L^{X}}(B, C(B)) \rightarrow C(B)=C(B) .
$$

Since $B \odot e_{L^{X}}(B, C(A)) \leq C(A)$, then

$$
B \leq \bigwedge_{A \in L^{X}}\left(e_{L^{X}}(B, C(A)) \rightarrow C(A)\right.
$$


Since $C_{M_{C}}(B)=\bigwedge_{A \in L^{X}}\left(e_{L^{X}}(B, C(A)) \rightarrow C(A) \in M_{C}\right.$, by (M2),

$$
\top=e_{L^{X}}(C(B), C(B)) \odot e_{X}\left(B, C_{M_{C}}(B)\right) \leq e_{X}\left(C(B), C_{M_{C}}(B)\right) .
$$

Hence $C(B) \leq C_{M_{C}}(B)$. Thus, $C_{M_{C}}=C$.

Let $M=\left\{A_{i} \mid i \in \Gamma\right\}$ be a fuzzy closure system. Then $C_{M}(A)=$ $\bigwedge_{i \in \Gamma}\left(e_{L^{X}}\left(A, A_{i}\right) \rightarrow A_{i}\right)$. Hence $M_{C_{M}}=\left\{\bigwedge_{i \in \Gamma}\left(e_{L^{X}}\left(A, A_{i}\right) \rightarrow A_{i}\right) \mid A \in L^{X}\right\}$. Let $A_{j} \in M$. Then $\bigwedge_{i \in \Gamma}\left(e_{L^{X}}\left(A_{j}, A_{i}\right) \rightarrow A_{i}\right)=e_{L^{X}}\left(A_{j}, A_{j}\right) \rightarrow A_{j}=A_{j}$. Let $k_{A}=\bigwedge_{i \in \Gamma}\left(e_{L^{X}}\left(A, A_{i}\right) \rightarrow A_{i}\right) \in M_{C_{M}}$. By Theorem 8(2), $k_{A} \in M$.

Theorem 21. Let $\left(X, e_{X}\right)$ be a fuzzy partial ordered set.

(1) If $(X, C)$ be a fuzzy closure operator, then $(X, C)$ is a fuzzy interior operator on $\left(X, e_{X}^{-1}\right)$ where $e_{X}^{-1}(x, y)=e_{X}(y, x)$.

(2) If $(X, I)$ be a fuzzy interior operator, then $(X, I)$ is a fuzzy closure operator on $\left(X, e_{X}^{-1}\right)$.

Theorem 22. Let $\left(L^{X}, e_{L^{X}}\right)$ be a fuzzy partial ordered set.

(1) If $\left(L^{X}, C\right)$ is a fuzzy closure operator on $L^{X}$, then $\left(L^{X}, C\right)$ is a fuzzy interior system on $\left(L^{X}, e_{L^{X}}^{-1}\right)$ where

$$
e_{L^{X}}^{-1}(A, B)=e_{L^{X}}(B, A)=e_{L^{X}}\left(A^{*}, B^{*}\right) .
$$

In particular, define $I(A)=C\left(A^{*}\right)^{*}$ and $I(A)=C\left(A^{*}\right)^{*}$. Then $(X, I)$ is a fuzzy interior operator on $\left(L^{X}, e_{L^{X}}\right)$.

(2) If $(X, I)$ is a fuzzy interior operator on $L^{X}$, then $(X, I)$ is a fuzzy closure operator on $\left(L^{X}, e_{L^{X}}^{-1}\right)$.

In particular, define $C(A)=I\left(A^{*}\right)^{*}$. Then $(X, C)$ is a fuzzy closure operator on $\left(L^{X}, e_{L^{X}}\right)$.

Proof. (1) Since $e_{L^{X}}^{-1}(A, B)=e_{L^{X}}(B, A)=e_{L^{X}}\left(A^{*}, B^{*}\right)$,

$$
\begin{aligned}
e_{L^{X}}\left(C(A)^{*}, A^{*}\right) & =e_{L^{X}}^{-1}(C(A), A)=e_{L^{X}}(A, C(A))=\top \\
e_{L^{X}}\left(C(A)^{*}, C(C(A))^{*}\right) & =e_{L^{X}}^{-1}(C(A), C(C(A))) \\
& =e_{L^{X}}(C(C(A)), C(A))=\top \\
e_{L^{X}}\left(A^{*}, B^{*}\right) & =e_{L^{X}}^{-1}(A, B)=e_{L^{X}}(B, A) \\
& \leq e_{L^{X}}(C(B), C(A))=e_{L^{X}}\left(C(A)^{*}, C(B)^{*}\right) .
\end{aligned}
$$

Put $A=A^{*}$. Then

$$
\begin{array}{cl}
e_{L^{X}}\left(C\left(A^{*}\right)^{*}, A\right) & =\top \\
e_{L^{X}}\left(C\left(A^{*}\right)^{*}, C\left(C\left(A^{*}\right)\right)^{*}\right) & =\top \\
e_{L^{X}}(A, B) & \leq e_{L^{X}}\left(C\left(A^{*}\right)^{*}, C\left(B^{*}\right)^{*}\right) .
\end{array}
$$


Put $I(A)=C\left(A^{*}\right)^{*}$. Then $I(I(A))=C\left(C\left(A^{*}\right)\right)^{*}(A)$. Thus

$$
\begin{aligned}
e_{L^{X}}(I(A), A) & =e_{L^{X}}(I(A), I(I(A)))=\top \\
e_{L^{X}}(A, B) & \leq e_{L^{X}}(I(A), I(A)) .
\end{aligned}
$$

(2) Since $e_{L^{X}}^{-1}(A, B)=e_{L^{X}}(B, A)=e_{L^{X}}\left(A^{*}, B^{*}\right)$,

$$
\begin{aligned}
e_{L^{X}}\left(A^{*}, I(A)^{*}\right) & =e_{L^{X}}^{-1}(A, I(A))=e_{L^{X}}(I(A), A)=\top \\
e_{L^{X}}\left(I(I(A))^{*}, I(A)^{*}\right) & \left.=e_{L^{X}}^{-1}(I(A)), I(A)\right) \\
& =e_{L^{X}}(I(A), I(I(A)))=\top \\
e_{L^{X}}\left(A^{*}, B^{*}\right) & =e_{L^{X}}^{-1}(A, B)=e_{L^{X}}(B, A) \\
& \leq e_{L^{X}}(I(B), I(A))=e_{L^{X}}\left(I(A)^{*}, I(B)^{*}\right) .
\end{aligned}
$$

Put $A=A^{*}$. Then

$$
\begin{array}{cl}
e_{L^{X}}\left(A, I\left(A^{*}\right)^{*}\right) & =\top \\
e_{L^{X}}\left(I\left(A^{*}\right)^{*}, I\left(I\left(A^{*}\right)\right)^{*}\right) & =\top \\
e_{L^{X}}(A, B) & \leq e_{L^{X}}\left(I\left(A^{*}\right)^{*}, I\left(B^{*}\right)^{*}\right) .
\end{array}
$$

Put $C(A)=I\left(A^{*}\right)^{*}$. Then $C(C(A))=I\left(I\left(A^{*}\right)\right)^{*}$. Thus

$$
\begin{aligned}
e_{L^{X}}(A, C(A)) & =e_{L^{X}}(C(C(A)), C(A))=\top \\
e_{L^{X}}(A, B) & \leq e_{L^{X}}(C(A), C(B)) .
\end{aligned}
$$

Theorem 23. The following statement are equivalent.

(1) An operator $I: L^{X} \rightarrow L^{X}$ is a fuzzy interior operator on $L^{X}$

(2) I satisfies (I1) and

(II) $\alpha \odot I(A) \leq I(\alpha \odot A)$ and $I(A) \leq I(B)$ for $A \leq B$.

(3) I satisfies (I1) and

(III) $I(\alpha \rightarrow A) \leq \alpha \rightarrow I(A)$ and $I(A) \leq I(B)$ for $A \leq B$.

Proof. (1) $\Rightarrow(2)$ If $A \leq B$, then $\top=e_{L^{X}}(A, B) \leq e_{L^{X}}(I(A), I(B))$. Thus, $I(A) \leq I(B)$. Since $\alpha \leq e_{L^{X}}(A, \alpha \odot A)$, we have $\alpha \leq e_{L^{X}}(A, \alpha \odot A) \leq$ $e_{L^{X}}(I(A), I(\alpha \odot A))$. Thus $\alpha \odot I(A) \leq I(\alpha \odot A)$.

$(2) \Rightarrow(1)$. Put $\alpha=e_{L^{X}}(A, B)$. By (II), $\alpha \odot I(A) \leq I(\alpha \odot A)=I\left(e_{L^{X}}(A, B) \odot\right.$ $A) \leq I(B)$. Hence $e_{L^{X}}(A, B) \leq e_{L^{X}}(I(A), I(B))$.

$(2) \Rightarrow(3)$ Since $\alpha \odot I(\alpha \rightarrow A) \leq I(\alpha \odot(\alpha \rightarrow A)) \leq I(A)$, then $I(\alpha \rightarrow A) \leq$ $\alpha \rightarrow I(A)$. 
$(3) \Rightarrow(2)$ Since $I(\alpha \rightarrow \alpha \odot A) \leq \alpha \rightarrow I(\alpha \odot A)$ iff $\alpha \odot I(\alpha \rightarrow \alpha \odot A) \leq$ $I(\alpha \odot A)$, we have

$$
\alpha \odot I(A) \leq \alpha \odot I(\alpha \rightarrow \alpha \odot A) \leq I(\alpha \odot A) .
$$

Theorem 24. The following statement are equivalent.

(1) An operator $C: L^{X} \rightarrow L^{X}$ is a fuzzy closure operator on $L^{X}$

(2) $C$ satisfies $(\mathrm{C} 1)$ and

(II) $\alpha \odot C(A) \leq C(\alpha \odot A)$ and $C(A) \leq C(B)$ for $A \leq B$.

(3) $C$ satisfies $(\mathrm{C} 1)$ and

(III) $C(\alpha \rightarrow A) \leq \alpha \rightarrow C(A)$ and $C(A) \leq C(B)$ for $A \leq B$.

Proof. (1) $\Rightarrow(3)$. (C1) If $A \leq B$, then $\top=e_{L^{X}}(A, B) \leq e_{L^{X}}(C(A), C(B))$. Thus, $C(A) \leq C(B)$.

(III) Since $\alpha \leq e_{L^{X}}(\alpha \rightarrow A, A)$, we have $\alpha \leq e_{L^{X}}(\alpha \rightarrow A, A) \leq e_{L^{X}}(C(\alpha \rightarrow$ $A), C(A))$. It implies $\alpha \odot C(\alpha \rightarrow A) \leq C(A)$. Thus $C(\alpha \rightarrow A) \leq \alpha \rightarrow C(A)$.

$(3) \Rightarrow(1)$. Put $\alpha=e_{L^{X}}(A, B)$. By (III), $C\left(e_{L^{X}}(A, B) \rightarrow B\right) \leq e_{L^{X}}(A, B) \rightarrow$ $C(B)$ implies $e_{L^{X}}(A, B) \leq C\left(e_{L^{X}}(A, B) \rightarrow B\right) \rightarrow C(B)$. Since $e_{L^{X}}(A, B) \odot A \leq$ $B$, then $A \leq e_{L^{X}}(A, B) \rightarrow B$. Since $C(A) \leq C\left(e_{L^{X}}(A, B) \rightarrow B\right)$, we have

$$
e_{L^{X}}(A, B) \leq C\left(e_{L^{X}}(A, B) \rightarrow B\right) \rightarrow C(B) \leq C(A) \rightarrow C(B) .
$$

Thus $e_{L^{X}}(A, B) \leq e_{L^{X}}(C(A), C(B))$.

$(3) \Rightarrow(2)$ Let $C$ be an operator satisfying (3). Since $C(\alpha \rightarrow \alpha \odot A) \leq \alpha \rightarrow$ $C(\alpha \odot A)$ iff $\alpha \odot C(\alpha \rightarrow \alpha \odot A) \leq C(\alpha \odot A)$, we have

$$
\alpha \odot C(A) \leq \alpha \odot C(\alpha \rightarrow \alpha \odot A) \leq C(\alpha \odot A) .
$$

$(2) \Rightarrow(3) \alpha \odot C(\alpha \rightarrow A) \leq C(\alpha \odot(\alpha \rightarrow A)) \leq C(A)$, then $C(\alpha \rightarrow A) \leq$ $\alpha \rightarrow C(A)$.

Theorem 25. (1) If $\mathcal{K}=\left\{A_{i} \mid i \in \Gamma\right\}$ is a fuzzy interior system on $L^{X}$, we define $I_{\mathcal{K}}: L^{X} \rightarrow L^{X}$ as $I_{\mathcal{K}}(A)=\bigvee\left\{A_{i} \mid A_{i} \leq A, A_{i} \in \mathcal{K}\right\}$. Then $I_{\mathcal{K}}(A)=\bigvee_{A_{i} \in \mathcal{K}}\left(e_{L^{X}}\left(A_{i}, A\right) \odot A_{i}\right)$ is a fuzzy interior operator on $X$.

(2) If $\mathcal{M}=\left\{A_{i} \mid i \in \Gamma\right\}$ is a fuzzy closure system on $L^{X}$, we define $C_{\mathcal{M}}: L^{X} \rightarrow L^{X}$ as $C_{\mathcal{M}}(A)=\bigwedge\left\{A_{i} \mid A \leq A_{i}, A_{i} \in \mathcal{M}\right\}$. Then $C_{\mathcal{M}}(A)=$ $\bigwedge_{A_{i} \in \mathcal{F}}\left(e_{L^{X}}\left(A, A_{i}\right) \rightarrow A_{i}\right)$ is a fuzzy closure operator on $L^{X}$. 
Proof. (1) Since $\bigvee_{i \in \Gamma}\left(e_{L^{X}}\left(A_{i}, A\right) \odot A_{i}\right) \leq A$ and $\bigvee_{i \in \Gamma} e_{L^{X}}\left(A_{i}, A\right) \odot A_{i} \in \mathcal{K}$, we have $\bigvee_{i \in \Gamma}\left(e_{L^{X}}\left(A_{i}, A\right) \odot A_{i}\right) \leq I_{\mathcal{K}}(A)$. Since $A_{i} \leq A, e_{L^{X}}\left(A_{i}, A\right) \odot A_{i}=A_{i}$. Hence $I_{\mathcal{K}}(A) \leq \bigvee_{i \in \Gamma}\left(e_{L^{X}}\left(A_{i}, A\right) \odot A_{i}\right)$. Thus, $I_{\mathcal{K}}(A)=\bigvee_{i \in \Gamma}\left(e_{L^{X}}\left(A_{i}, A\right) \odot A_{i}\right)$. By Theorem 18(2), $I_{\mathcal{K}}(A)=k_{A}$ is a fuzzy interior operator.

(2) Since $A \leq \bigwedge_{i \in \Gamma}\left(e_{L^{X}}\left(A, A_{i}\right) \rightarrow A_{i}\right)=m_{A} \in \mathcal{M}$, we have $C_{\mathcal{M}}(A) \leq$ $\bigwedge_{i \in \Gamma}\left(e_{L^{X}}\left(A, A_{i}\right) \rightarrow A_{i}\right)$. For $A \leq A_{i}$ with $A_{i} \in \mathcal{M}$, then $e_{L^{X}}\left(A, A_{i}\right) \rightarrow A_{i}=$ $\mathrm{\top} \rightarrow A_{i}=A_{i}$. Hence $\bigwedge_{A_{i} \in \mathcal{M}}\left(e_{L^{X}}\left(A, A_{i}\right) \rightarrow A_{i}\right) \leq C_{\mathcal{M}}(A)$. By Theorem 20(2), $C_{\mathcal{M}}(A)=\bigwedge_{A_{i} \in \mathcal{M}}\left(e_{L^{X}}\left(A, A_{i}\right) \rightarrow A_{i}\right)$.

Example 26. Let $(L=[0,1], \odot)$ be a residuated lattice defined by

$$
x \odot y=(x+y-1) \vee 0, x \rightarrow y=(1-x+y) \wedge 1
$$

Let $X=\{a, b\}$ and $A \in L^{X}$ as follows: $A(a)=0.6, A(b)=0.8$.

Let $\mathcal{M}=\{\alpha \rightarrow A \mid \alpha \in L\}$ and $\mathcal{K}=\{\alpha \odot A \mid \alpha \in L\}$ be given.

(1) $\mathcal{M}$ is a fuzzy closure system from the following statements. For each $D \in L^{X}$,

$$
\begin{aligned}
C_{\mathcal{M}}(D)=m_{D} & =\bigwedge_{\alpha \in L}\left(e_{L^{X}}(D, \alpha \rightarrow A) \rightarrow(\alpha \rightarrow A)\right. \\
& =\bigwedge_{\alpha \in L}\left(\left(\alpha \rightarrow e_{L^{X}}(D, A)\right) \rightarrow(\alpha \rightarrow A)\right. \\
& \geq e_{L^{X}}(D, A) \rightarrow A, \\
C_{\mathcal{M}}(D)=m_{D} & =\bigwedge_{\alpha \in L}\left(e_{L^{X}}(D, \alpha \rightarrow A) \rightarrow(\alpha \rightarrow A)\right. \\
& \leq\left(\top \rightarrow e_{L^{X}}(D, A)\right) \rightarrow(\top \rightarrow A) \\
& \leq e_{L^{X}}(D, A) \rightarrow A .
\end{aligned}
$$

Hence $C_{\mathcal{M}}(D)=m_{D}=e_{L^{X}}(D, A) \rightarrow A \in \mathcal{M}$. Moreover,

$$
M_{C_{\mathcal{M}}}=\left\{e_{L^{X}}(D, A) \rightarrow A \mid D \in L^{X}\right\}=\{\alpha \rightarrow A \mid \alpha \in L\}=\mathcal{M} .
$$

From Theorem 14(1), $\mathcal{K}_{M}=\left\{\alpha \odot A^{*} \mid \alpha \in L\right\}$ is a fuzzy interior system where $A^{*}(a)=0.4, A^{*}(b)=0.2$. Moreover,

$$
\begin{aligned}
I_{\mathcal{K}_{M}}(D)=k_{D} & =\left(m_{D^{*}}\right)^{*}=\left(e_{L^{X}}(A, D) \rightarrow A\right)^{*} \\
& =e_{L^{X}}\left(A^{*}, D\right) \odot A^{*} .
\end{aligned}
$$

$(2) \mathcal{K}$ is a fuzzy interior system from the following statements. For each $D \in L^{X}$,

$$
\begin{aligned}
I_{\mathcal{K}}(D)=k_{D} & =\bigvee_{\alpha \in L}\left(e_{L^{X}}(\alpha \odot A, D) \odot(\alpha \odot A)\right. \\
& =\bigwedge_{\alpha \in L}\left(\left(\alpha \rightarrow e_{L^{X}}(A, D)\right) \odot \alpha\right) \odot A \\
& \leq e_{L^{X}}(A, D) \odot A \\
I_{\mathcal{K}}(D)=k_{D} & =\bigvee_{\alpha \in L}\left(e_{L^{X}}(\alpha \odot A, D) \odot(\alpha \odot A)\right) \\
& \geq e_{L^{X}}(\top \odot A, D) \odot(\top \odot A) \\
& =e_{L^{X}}(A, D) \odot A .
\end{aligned}
$$


Hence $I_{\mathcal{K}}(D)=k_{D}=e_{L^{X}}(A, D) \odot A \in \mathcal{K}$. Moreover,

$$
K_{I_{\mathcal{K}}}=\left\{e_{L^{X}}(A, D) \odot A \mid D \in L^{X}\right\}=\{\alpha \odot A \mid \alpha \in L\}=\mathcal{K} .
$$

From Theorem $14(2), \mathcal{M}_{K}=\left\{\alpha \rightarrow A^{*} \mid \alpha \in L\right\}$ is a fuzzy closure system where $A^{*}(a)=0.4, A^{*}(b)=0.2$. Moreover,

$$
\begin{aligned}
C_{\mathcal{M}_{K}}(D)=m_{D} & =\left(k_{D^{*}}\right)^{*}=\left(e_{L^{X}}\left(A, D^{*}\right) \odot A\right)^{*} \\
& =e_{L^{X}}\left(A, D^{*}\right) \rightarrow A^{*}
\end{aligned}
$$

Let $B(a)=0.7, B(b)=0.5$ be given.

(3) We can find the least element $\alpha=0.9$ such that $B \leq \alpha \rightarrow A$. Hence

$$
C_{\mathcal{M}}(B)=0.9 \rightarrow A=(0.7,0.9)
$$

(4) We can find the greatest element $\alpha=0.7$ such that $\alpha \odot A \leq B$. Hence

$$
I_{\mathcal{K}}(B)=0.7 \odot A=(0.3,0.5) .
$$

\section{References}

[1] R. Bělohlávek, Fuzzy Galois connection, Math. Log. Quart., 45 (2000), 497-504.

[2] R. Bělohlávek, Fuzzy closure operator, J. Math. Anal. Appl., 262 (2001), 473-486.

[3] R. Bělohlávek, Fuzzy Relational Systems, Kluwer Academic Publisher, New York (2002).

[4] G. Georgescu, A. Popescue, Non-commutative Galois connections, Soft Computing, 7(2003), 458-467.

[5] G. Georgescu, A. Popescue, Non-dual fuzzy connections, Arch. Math. Logic 43(2004), 1009-1039.

[6] L. Guo, G.Q. Zhang, Q. Li, Fuzzy closure systems on L-ordered sets, Math. Log. Quart. 57(3) (2011), 281-291.

[7] U. Höhle, E. P. Klement, Non-classical logic and their applications to fuzzy subsets, Kluwer Academic Publisher, Boston (1995). 
[8] E. Turunen, Mathematics Behind Fuzzy Logic, A Springer-Verlag Co., (1999).

[9] W. Yao, L,-X, Lu, Fuzzy Galois connections on fuzzy posets, Math. Log. Quart. 55(1) (2009), 105-112. 